\title{
Bonding Character and Formation Energy of Point Defects of He and Vacancy on (001) Surface of bcc Iron by First Principle Calculations
}

\author{
Jun $C A I^{\dagger}$ and Daogang $L U$ \\ School of Nuclear Science and Engineering, North China Electric Power University, Beijing 102206, China \\ [Manuscript received 23 August 2011, in revised form 28 December 2011] \\ (C) The Chinese Society for Metals and Springer-Verlag Berlin Heidelberg
}

\begin{abstract}
The structure and energy of He impurities and vacancy on (001) surface of bcc iron are investigated by an ab initio method. Three cases for stabilities of a He atom at the surface are found: some of He atoms at surface atomic layers (SAL) relax into vacuum gap; some of surface He atoms at octahedral interstitial site relax into more stable tetrahedral interstitial site; some of surface He atoms still stay at tetrahedral interstitial site. The un-stability of the $\mathrm{He}$ atom at the surface system can be explained by deformation mechanism of charge densities and electronic densities of states. It is found that formation energy of the point defects from the topmost SAL to bulk-like atomic layer increase gradually, for example, the formation energies of a monovacancy at the first five topmost SALs are equal to $0.33,1.56,2.04,2.02$ and $2.11 \mathrm{eV}$, respectively. The magnetic moments of Fe atoms in the surface atomic layers are also calculated.
\end{abstract}

KEY WORDS: ab initio calculations; Single point defects; bcc Fe (001) surface; Electronic structure

\section{Introduction}

Irradiation with a few tens of $\mathrm{MeV}$ neutrons leads to the formation of displacement cascades in structural materials used in nuclear reactor, and the cascades will induce vacancies, self-interstitial atoms (SIA), and fission products such as $\mathrm{H}$ and $\mathrm{He}$ in the structural materials. The defects may diffuse and aggregate in the materials, and lead to the degradation of the mechanical properties. He is known to be a main fission production and may induce certain micro-structural changes in the ferritic materials, such as the formation of the He bubbles. The He bubbles are believed to give rise to hardening, embrittlement, and swelling of the materials. The interaction of $\mathrm{He}$ with other defects such as vacancies and SIA is also required to be investigated in order to completely understand the effect of He on the mechanical properties of structural materials used in the nuclear reactor.

\footnotetext{
† Corresponding author. Prof., Ph.D.; Tel: +86 1051963854 ; E-mail address: caijun@ncepu.edu.cn (Jun CAI)
}

DOI: $10.1007 / \mathrm{s} 40195-011-0504-\mathrm{z}$
The effect of He on the mechanical properties of low activation ferrite-based steels has been studied extensively both theoretically and experimentally. Nevertheless, the issue has still not been completely resolved up to now ${ }^{[1-3]}$.

First principle calculation is a useful tool to study the basic properties of defect. For example, by first principle calculations, Terentyev et al. ${ }^{[2]}$ confirmed the existence of unexpected configurations of selfinterstitial clusters in bcc iron, formed by nonparallel dumbbells. The properties of these configurations provide a new picture, which clarified a longstanding debate on the properties of SIA clusters in iron. Fu and Willaime ${ }^{[4]}$ performed ab initio calculations of He in bcc iron and found that the substitutional and interstitial configurations of He have similar stabilities. Interstitial He atoms are predicted to have attractive interactions and a very low migration energy, suggesting that He bubbles can form at low temperature. The relative stabilities of the various He solution sites were also revealed by recent ab initio calculations $^{[5]}$. The studies found that the tetrahedral position is energetically more favorable for He inter- 
stitial in bcc iron than the octahedral site ${ }^{[4,5]}$. Using DFT method, Domain ${ }^{[6]}$ studied systematically SIA, foreign interstitial defects $(\mathrm{C}, \mathrm{H}$, and $\mathrm{He})$ in different matrix elements (Fe and $\mathrm{Cr}$ ). In his works some substitutional elements $(\mathrm{Cu}, \mathrm{Ni}, \mathrm{Mn}, \mathrm{Cr}$, and $\mathrm{P})$ in bcc Fe were also presented. Domain found that in bcc Fe, solute atom interaction with vacancy is not directly linked to elastic interaction due to solute size effect, but chemical interaction seems to play an important role. It was also confirmed that the $\langle 110\rangle$ SIA in bcc $\mathrm{Fe}$ is the most stable configuration. Recently, by electronic structure calculations Seleskaia et al. ${ }^{[7]}$ studied the relaxation, formation and binding energies of small He cluster in bcc iron. Although these calculations presented some properties of He atom in bulk of bcc iron, to our best knowledge, there are few theoretical reports about the interaction of He atom with surface defects of bcc iron, such as the interaction of He atom with surface of bcc iron. On the experimental aspect, $\mathrm{Xu}$ and Wirth ${ }^{[3]}$ recently also studied He desorption on bcc single-crystalline and estimated the average activation energies of He on bcc Fe. However, the underlying microscopic mechanism for all desorption groups are not entirely clear. On the other hand, Cai and Phillpot ${ }^{[8]}$ showed that the atoms in the surface might be different from ones in bulk. Thus it is of great interest to understand the characters of the bonding and formation of He atom in the surface of bcc iron at the atom scale. This kind of characters will play a key role in the diffusion and transferring of the atoms on the surface of materials. In this paper, the structure and energy of He impurities and vacancy on (001) surface of bcc iron are studied and the formation mechanism of He bubble is also discussed.

\section{Theory and Method}

In this paper we study the electronic structure of He atom on surface atomic layers (SAL) of bcc Fe using a modern ab initio pseudo-potential method ${ }^{[9,10]}$. We first optimize lattice parameters of bulk of bcc Fe and then perform a calculation for the surface system without any impurities. Finally, we carry out the numerical simulations once again for the surface system by introducing a single point defect. The single point defects include various kinds of point defects, such as a monovacancy instead of a Fe atom, a He atom placed at a tetrahedral interstitial site (TIS) or at an octahedral interstitial site (OIS), or at a substitutional site (SS) of Fe atom. These point defects are introduced into the different SALs of bcc iron. We calculate their formation energies and electronic structures. The calculations are carried out within the framework of density function theory ${ }^{[11,12]}$ using a modern ab initio pseudo-potential method ${ }^{[9,10]}$. The approach that is implemented in the Vienna ab initio software package (VASP) is based on a finite-temperature local-density approximation (with the free energy as variational quantity ${ }^{[9-13]}$, an ex- act evaluation of the instantaneous electronic ground state at each ion-step using efficient matrix diagonalization schemes ${ }^{[14]}$ and an efficient Pulay mixing ${ }^{[15]}$. These techniques avoid all problems occurring in the original Car-Parrinello method ${ }^{[16]}$ which is based on the simultaneous integration of electronic and ionic equations of motion. The interaction between the ions and electrons can be described by using either ultrasoft Vanderbilt pseudopotentials ${ }^{[17]}$ or the projector augmented wave method ${ }^{[18]}$. Here the projector augmented wave method is used. Forces and stress can be easily calculated with VASP and are used to relax atoms into their instantaneous ground state. The more detail about VASP can be found in Ref.13-16. In present calculations, the exchangecorrelation function of Ceperly and Alder parameterized by Perdew and Zunger ${ }^{[19]}$ with the generalized gradient approximation ${ }^{[20]}$ is implemented in VASP code $^{[9,10]}$. A conjugate-gradient algorithm is used to relax the ions into instantaneous ground-state ${ }^{[9,10]}$. In these calculations, Monkhorst-Pack ${ }^{[21]} k$-point sampling density of $5 \times 5 \times 5$ is sufficient to obtain the accurate lattice parameters and the total energy of the bulk solid with a $3 \times 3 \times 3$ bcc unit cells consisting of $54 \mathrm{Fe}$ atoms, and a tetrahedron method is used with Bloechl correction. For surface calculations the $5 \times$ $5 \times 1 k$-point sampling density was used. The surface system is a slab model that consists of a point defect, a vacuum gap with thickness of $1.25 \mathrm{~nm}$, and $13 \mathrm{Fe}$ atomic layers with a $3 \times 3 \times 13$ bcc unit cells along the $x, y$, and $z$ direction, respectively. The plane wave cut-off energy of $348.28 \mathrm{eV}$ is used in combination with high precision calculations of VASP. The relaxation of electronic degrees of freedom is stopped if the total energy change of the system between two steps is smaller than $10^{-5} \mathrm{eV}$, while the coordinates of the atom are fully relaxed when the total energy between two ionic relaxation steps is smaller than $10^{-4} \mathrm{eV}$.

\section{Results and Discussions}

(001) surface of Fe is often studied experimentally and theoretically ${ }^{[22,23]}$. Here we also choose it to study the interaction of single point defects with the surface. Fig. 1 is the surface system for the surface calculations. In the left panel of the figure, the surface system is built by a slab model that consists of $3 \times 3 \times 13$ bcc unit cells of bcc Fe along the $x, y$, and $z$ crystallographic directions, respectively, and a vacuum gap of $1.25 \mathrm{~nm}$. For an eye-guide, in the right panel we show a $1 \times 1 \times 2$ unit cell taking from the first three topmost Fe atomic surface layers, and at the cell the sites of some kinds of point defects are labeled by numbers from 1 to 7 . In the first topmost surface atomic layers (SAL) there are three point defect sites, where point defects 1 and 3 are at octahedral interstitial site (OIS) and they are not equivalent to each other due to breakdown of symmetry in surface, and site 2 is a tetrahedral interstitial site (TIS). The same 


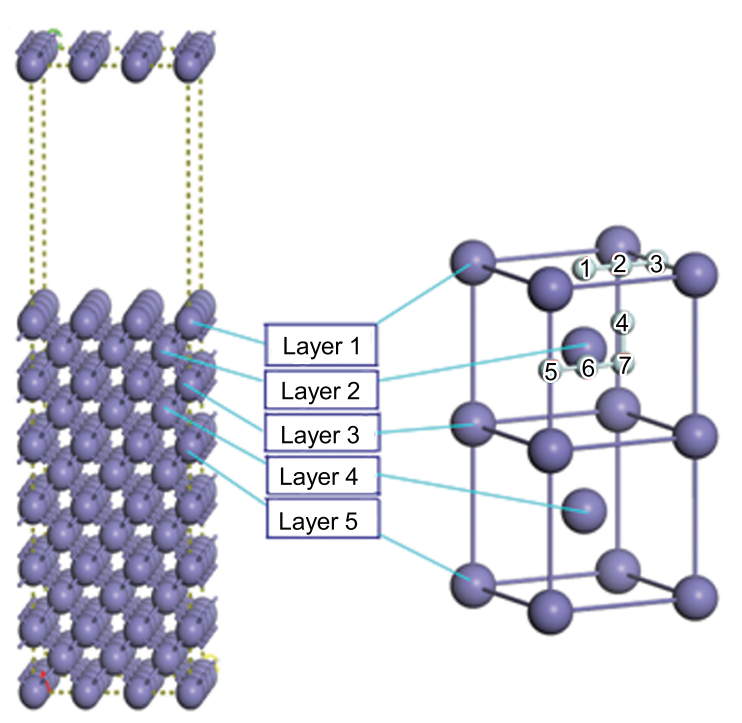

Fig. 1 Computational supercell, the surface system is modeled as a slab model and consists of $3 \times 3 \times 13$ bcc unit cells of bcc Fe in $x, y$, and $z$ crystallographic directions and a vacuum gap with thickness of $1.25 \mathrm{~nm}$. The surface system is shown in the left panel of the figure. In the right panel some sites of the point defects are labeled by number 1 to 7 , where in the first topmost surface atomic layer (layer 1) there are three point defect sites, and sites 1 and 3 are OISs, and site 2 is a TIS. In the other layers there are also similar point defect sites. A monovacancy might be produced by removing a $\mathrm{Fe}$ atom on these surface atomic layers (SALs). Atoms at the first five topmost and bottommost SALs are allowed to fully relax. The other atomic layers are fixed to simulate bulk-like surrounding

as in the first topmost SAL, in the second topmost SAL three similar point defects are also shown, and point defects 5 and 7 are at OISs, point defect 6 is at TIS. Between the first and the second topmost SALs there is a TIS point defect 4 . On the other layers there are similar point defect sites, which are not shown here. A monovacancy can be produced by removing a Fe atom on the different SALs. For convenience, we mark the first topmost SAL as layer 1, the second one as layer 2, and so on. Atoms at the first five topmost and bottommost SALs are allowed to fully relax. The other atomic layers are fixed to simulate bulk-like surrounding. We calculate the formation energy of He atom at TIS, or at OIS, and or at substitutional site (SS), as well as the formation energy of monovacancy on different (001) topmost SAL of bcc iron. From Fig. 1 it can be found that on the each SAL there are two kinds of OIS. Symbols $O_{n \perp}$ and $O_{n / /}$ to expresses the He atom at the two kinds of OISs at layer $n$. The former denotes the impurity with two nearest neighboring Fe atoms along vertical $(z)$ direction (sites 1 and 5 in Fig. 1) and the latter does the one with two nearest neighboring Fe atoms along horizontal ( $x$ or $y$ ) direction (see, for instants, sites 3 and 7 in Fig. 1). We use $T_{n}, S_{n}$, and $V_{n}$ to denote He atom at TIS (sites 2 and 6 in Fig. 1), SS at layer $n$, and a monovacancy at layer $n$, respectively. $T_{n / n+1}$ denotes a tetrahedral interstitial He atom on between layer $n$ and layer $n+1$ (site 4 in Fig. 1). For convenience, the formation energy of these point defects are labeled as $E_{O_{n} \perp}^{\mathrm{f}}, E_{O_{n} / /}^{\mathrm{f}}, E_{T_{n}}^{\mathrm{f}}, E_{S_{n}}^{\mathrm{f}}$, and $E_{V_{n}}^{\mathrm{f}}$, $E_{T_{n / n+1}}^{\mathrm{f}}$, respectively. Note that in these symbols we use subscript $n=\infty$ to denote the formation energy of a point defect in a bulk. $M_{n}$ is used to denote magnetic moment of Fe atom on layer $n$.

\subsection{Site occupancy of $\mathrm{He}$ in bcc Fe}

Firstly, we construct a supercell of bcc iron with $54 \mathrm{Fe}$ atoms. We optimize the lattice constant of bcc iron using VASP code ${ }^{[9,10]}$. In this calculations spin polarization is considered and $5 \times 5 \times 5 k$-point sampling density is used. We obtain a lattice parameter of bcc Fe to be equal to $0.28302 \mathrm{~nm}$. The lattice constant is in agreement with the experimental value of 0.2866 and the theoretical value of 0.2880 in previous calculations $^{[4]}$. We also calculate site occupancy of $\mathrm{He}$ atom in the supercell. The relative stability of various He occupied sites is determined by the sequence of their formation energies. For a bulk supercell containing $N$ Fe atoms and one He atom, the point defect formation energy, $E_{\text {defect }_{\infty}}^{\mathrm{f}}$, is calculated by

$$
E_{\text {defect }_{\infty}}^{\mathrm{f}}=E_{N \mathrm{Fe}+\mathrm{He}}-N E_{\mathrm{Fe}}-E_{\mathrm{He}}
$$

where $E_{N \mathrm{Fe}+\mathrm{He}}, E_{\mathrm{Fe}}, E_{\mathrm{He}}$ are the energies of the $\mathrm{Fe}+\mathrm{He}$ supercell, per Fe atom in a perfect bcc bulk and an isolated He atom, respectively. It is noted that $N$ is one less for the substitutional case compared with the interstitial case. The formation energies of different interstitial sites, such as octahedral interstitial, tetrahedral interstitial, and substitutional site, as well as monovacancy are calculated and listed in table 1 . He atom at the substitutional site is found to be energetically favorable structure over the structure of He atom in tetrahedral and octahedral interstitial sites. From the table it can be seen that the He atom at TIS of the bcc Fe is a more favorable structure in energetics over $0.20 \mathrm{eV}$ than at octahedral site. The formation energy of the former is calculated to be $4.56 \mathrm{eV}$ and the latter to be $4.76 \mathrm{eV}$. The vacancy formation energy and the formation energy of He atom at TIS or OIS of bcc Fe have been studied by ab initio calculations. In the previous calculations, $\mathrm{Zu}$ et al. ${ }^{[24]}$ gave the formation energy of He atom as TIS atom and OIS atom to be 4.49 and $4.68 \mathrm{eV}$, respectively. $\mathrm{Fu}$ and Willaime ${ }^{[4]}$ reported the results of 4.39 and $4.57 \mathrm{eV}$. Recently Zhang et al. ${ }^{[25]}$ present the corresponding results of 4.41 and $4.58 \mathrm{eV}$. As shown in Table 1 , the present results are in agreement with the previous theoretical works and experimental results. The formation energy of monovacancy is calculated to be equal to $2.15 \mathrm{eV}$ and is in good agreement with experimental data of $(2.0 \pm 0.2) \mathrm{eV}$ and the previous 
Table 1 The formation energies of single point defect in (001) surface and bulk of bcc Fe. $E_{T n}^{f}, E_{O n \perp}^{f}, E_{S n}^{f}$, and $E_{\mathrm{Vn}}^{f}$ express the formation energy of He atom at TIS, OIS, SS of (001) SALs of bcc Fe, and as well as the formation energy of monovacancy on different (001) SALs, respectively. Subscript $n$ expresses the point defects at the $n^{\text {th }}$ topmost SAL (i.e., layer $n$, see Fig. 1). $E_{T n / n+1}^{f}$ notes the formation energy of He atom at a TIS between layer $n$ and layer $n+1$. $M M A_{n}$ is used to expresses magnetic moment of Fe atom on layer $\mathrm{n}$ of the surface system without any impurities. Note in these symbols we use subscript $n=\infty$ to express the formation energy of a point defect or magnetic moment of Fe atom in a bulk.

\begin{tabular}{ccccccc}
\hline Defect site & $E_{V n}^{\mathrm{f}} / \mathrm{eV}$ & $E_{S n}^{\mathrm{f}} / \mathrm{eV}$ & $E_{T n}^{\mathrm{f}} / \mathrm{eV}$ & $E_{T n / n+1}^{\mathrm{f}} / \mathrm{eV}$ & $E_{O n \perp}^{\mathrm{f}} / \mathrm{eV}$ & $M M A_{n} / \mu_{\mathrm{B}}$ \\
\hline Layer 1 & 0.33 & - & - & - & - & 2.875 \\
Layer 2 & 1.56 & - & - & 4.28 & 4.42 & 2.238 \\
Layer 3 & 2.04 & 3.81 & 4.31 & 4.43 & 4.44 & 2.320 \\
Layer 4 & 2.02 & 3.88 & 4.47 & 4.46 & 4.66 & 2.137 \\
Layer 5 & 2.11 & 4.21 & 4.56 & 4.51 & 4.71 & 2.120 \\
Bulk & $2.15\left(2.07^{[4]}\right.$, & $4.25\left(4.13^{[25]}\right)$ & $4.56\left(4.35^{[6]}\right.$, & - & $4.76\left(4.57^{[6]}\right.$, & $2.16\left(2.31^{[4]}\right.$, \\
& $\left.1.93^{[6]}, 2.16^{[25]}\right)$ & & $\left.4.41^{[25]}\right)$ & & $\left.4.58^{[25]}\right)$ & $\left.2.32^{[25]}, 2.22^{[26]}\right)$ \\
\hline
\end{tabular}

theoretical value of 2.07 and $2.16 \mathrm{eV}^{[4,25]}$. The magnetic moment of $\mathrm{Fe}$ atom in bulk is calculated to $2.16 \mu \mathrm{B}$ and is in agreement with the experimental value of $2.22 \mu \mathrm{B}^{[26]}$. We perform further calculations for site occupancy of He atom in (001) surface of bcc iron.

\subsection{Site occupancy of He atom in (001) surface of bcc Fe}

In present calculations for (001) surface system of bcc Fe, the formation energy of monovacancy at layer $n$, and the formation energies of a He atom at SS, or TIS, and or OIS of layer $n$ are written as:

$$
\begin{gathered}
E_{V n}^{\mathrm{f}}=\left(E_{V_{n}}^{\mathrm{T}}+\mu_{\mathrm{Fe}}\right)-E_{\mathrm{surf}}^{\mathrm{T}} \\
E_{S n}^{\mathrm{f}}=E_{S_{n}}^{\mathrm{T}}+\mu_{\mathrm{Fe}}-E_{\text {surf }}^{\mathrm{T}}-E_{\mathrm{He}} \\
E_{T n}^{\mathrm{f}}=E_{T_{n}}^{\mathrm{T}}-E_{\text {surf }}^{\mathrm{T}}-E_{\mathrm{He}} \\
E_{O n}^{\mathrm{f}}=E_{O_{n}}^{\mathrm{T}}-E_{\text {surf }}^{\mathrm{T}}-E_{\mathrm{He}}
\end{gathered}
$$

respectively, where $\mu_{\mathrm{Fe}}=E_{\mathrm{Fe}}, E_{\mathrm{surf}}^{\mathrm{T}}$ is the total energy of the surface system without any point defect, $E_{\mathrm{V}_{\mathrm{n}}}^{\mathrm{T}}$ is the total energy of a surface system with a monvacancy at the $n^{\text {th }} \mathrm{SAL}$ (layer $n$ ), $E_{\mathrm{S}_{\mathrm{n}}}^{\mathrm{T}}, E_{\mathrm{T}_{\mathrm{n}}}^{\mathrm{T}}$, and $E_{\mathrm{O}_{\mathrm{n}}}^{\mathrm{T}}$ are the total energy of a surface system with a He atom at SS, TIS, and OIS of layer $n$, respectively. If the superscript $\mathrm{T}$ is replaced by $\mathrm{f}$, then they denote the formation energy of corresponding point defects. The numerical results of formation energies of these point defects are listed in Table 1. From Table 1 , it can also be seen that the formation energy for the same kind of point defect is changed with its site at different SAL. From the topmost SAL to bulk-like atomic layer the formation energies increase gradually. This indicates that all kinds of the point defects considered have great trend to aggregate to the topmost SAL. From the Table 1 it can also be see that the relative stability of the point defects at a deeper
SAL $(n>2)$ is in the order of monovacancy, SS, TIS, and OIS. The sequence of stability of these defects in each of the deeper SALs is the same as those in bulk.

In present calculations it is also found that all kinds of He atom point defects at the first topmost SAL can escape from the SAL and enter into the vacuum gap directly by full relaxation of atom structure. He atom at substitutional and tetrahedral interstitial sites on the second topmost SAL is also found to be unstable and can escape from the SAL and enter into the vacuum gap directly. In other word, the point defects entering into the vacuum gap directly by atom relaxation include $\mathrm{He}$ atom at $S_{1}, S_{2}, T_{1}, T_{2}, T_{1 / 2}$,

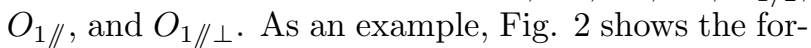
mation energy of He impurity atom to locate initially

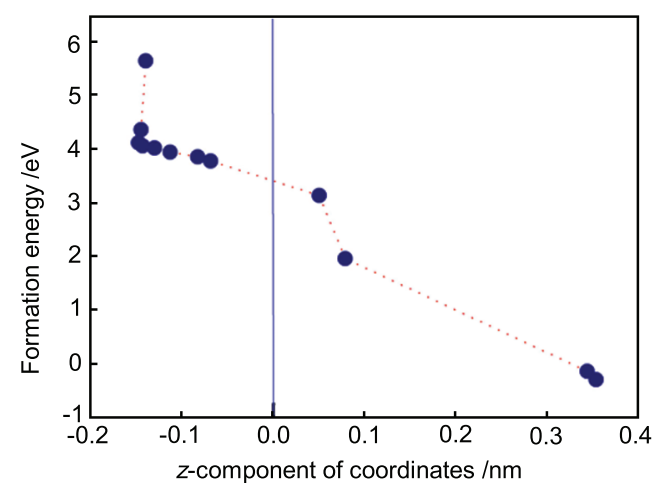

Fig. 2 The formation energy of He impurity atom as a function of the $z$-component of its coordinates, where the vertical blue line indicates that the position of the first topmost surface atomic layer (SAL) of the clean surface system locates at zero point of $z$-component coordinates. The short dash red line is for an eyes guide. Here He atom is placed at the position of $T_{2}$ (tetrahedral interstitial site at the second topmost SAL) initially, then by atom relaxation, the He atom does not meet any potential barrier, and it moves across the first topmost SAL and finally goes into the vacuum gap 
at $T_{2}$ as a function of the $z$-component of its coordinates during atom relaxation, where the vertical blue line at zero indicates $z$-component position of the first topmost SAL of the clean surface system. The short dash red line is for an eye-guide. Here He atom is placed at the position of $T_{2}$ (TIS at the second topmost SAL) initially, then by atom relaxation, the formation energy reduces with increasing $z$-component of the coordinate of the point defect. This indicates that during atom relaxation the $\mathrm{He}$ atom does not meet any potential barrier and "goes" across the topmost SAL, and ultimately it enters into the vacuum gap. From Fig. 2 it can also be seen that when the atom arrives at the vacuum gap and attains a height of about $0.35 \mathrm{~nm}$ relative to the first topmost SAL, the formation energy reduces to be slightly less than
$0 \mathrm{eV}$.

It is also find that the other some $\mathrm{He}$ atoms as point defects which are located initially at $O_{n / /}$ $(2 \leq n \leq 5)$ are unstable energetically and change into corresponding TIS defects by atom relaxation, i.e., $O_{2 / /} \rightarrow T_{2 / 3}, O_{3 / /} \rightarrow T_{2 / 3}, O_{4 / /} \rightarrow T_{4 / 5}$, and $\mathrm{O}_{5 / /} \rightarrow T_{5 / 6}$. Present numerical results shows that except for these unstable point defects mentioned above, all the other point defects are stable and are kept at their original defect sites during full relaxation of atom structure.

The un-stability of He atom as an impurity in the (001) SALs can be understood by plotting their deformation charge densities $(D)$ and electronic density of states (DOS). As an example, Fig. 3 shows the deformation charge densities for six cases corresponding
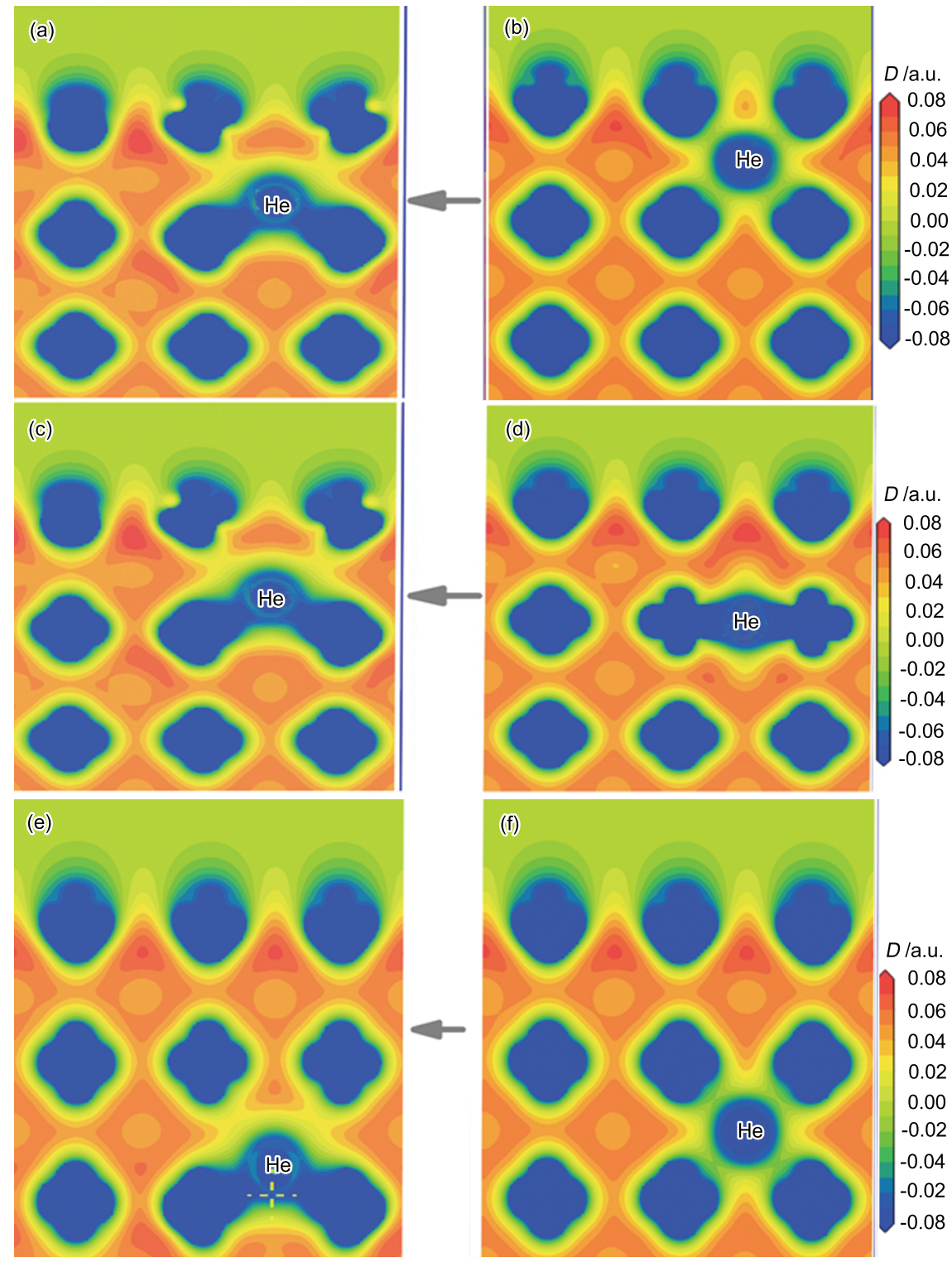

Fig. 3 The deformation charge density $(D)$ maps in (100) plane: (a), (c), and (e) correspond to the systems with a He atom initially placed at $O_{2 / /}, O_{3 / /}$, and $O_{4 / /}$ sites, respectively; (b), (d), and (f) correspond to the relaxed systems from (a), (c), and (e), respectively, where the He atom is relaxed into $T_{2 / 3}$, $T_{2 / 3}$, and $T_{4 / 5}$ sites, respectively. In Fig. 3 , the He atom is marked by its element symbol, the other sites with dark blue are Fe atoms at layer 1, layer 3, and layer 5, respectively, and the larger parts with green denote vacuum gap 
to the He atom at $O_{n / /}(n=2,3,4)$ and at $T_{n / n+1}$ $(n=2,2,4)$. For the same six cases the corresponding DOS is shown in Fig. 4. The deformation charge densities are plotted at on (100) plane for the surface system. The case of He atom at $O_{n / /}(n=2,3,4)$ corresponds to Fig. 3(b), (d) and (f), respectively. By a full relaxation of atom structure using VASP code, the He atom at $O_{n / /}(n=2,3,4)$ enters into respective site $T_{n / n+1}(n=2,2,4)$. For the three TIS cases their deformation charge density maps are plotted in Fig. 3(a), (c) and (e). From Fig. 3 it can be seen that the breakdown of symmetry by the surface leads to a vertical direction imbalance distribution of electronic density around He atom at $O_{n / /}$ site (see (b), (d), and (f)). The imbalance distribution should produce a force to "push" He atom into a $T_{n / n+1}$ site, and thus an equilibrium distribution of the electronic density is recovered (see Fig. 3(a), (c) and (e)). From Fig. 3 it can be also seen that between $\mathrm{He}$ atom and its two first nearest neighboring Fe atoms the charge
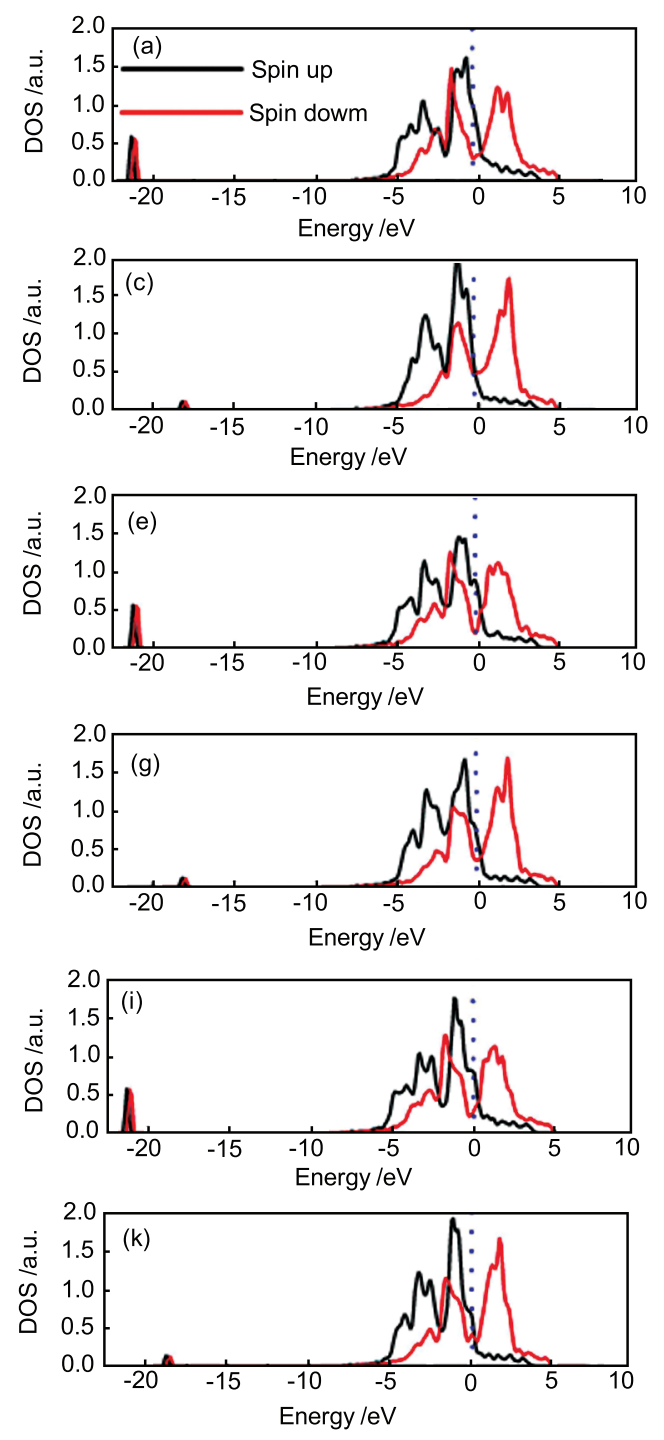

densities are less than the charge densities of overlapping by their respective isolated atoms. This indicates that it is difficult to form a stronger bond between $\mathrm{He}$ atom and Fe atom in the bcc Fe. At the same time this indicates that electronic cloud around He atom is reduced. It is well known that two He atoms with two electrons in their respective atomic orbital cannot form a stable molecular, while if each of them has only one electron a stable molecular of $\mathrm{He}$ can be formed. As the similar reason, if electronic cloud around He atom is reduced, as shown in Fig. 3, it perhaps is helpful to form a weak-link between the two He atoms. If it is true, this can be a reason that in bcc Fe, He atoms may form He bubble. Of course, the un-stability (as shown in present work) and the positive formation energy (as listed in Table 1) of He impurity in bcc Fe should be greatly helpful to form a He bubble. The van der walls interaction between He atoms might be also one of factors to provide an attractive force between the He atoms.
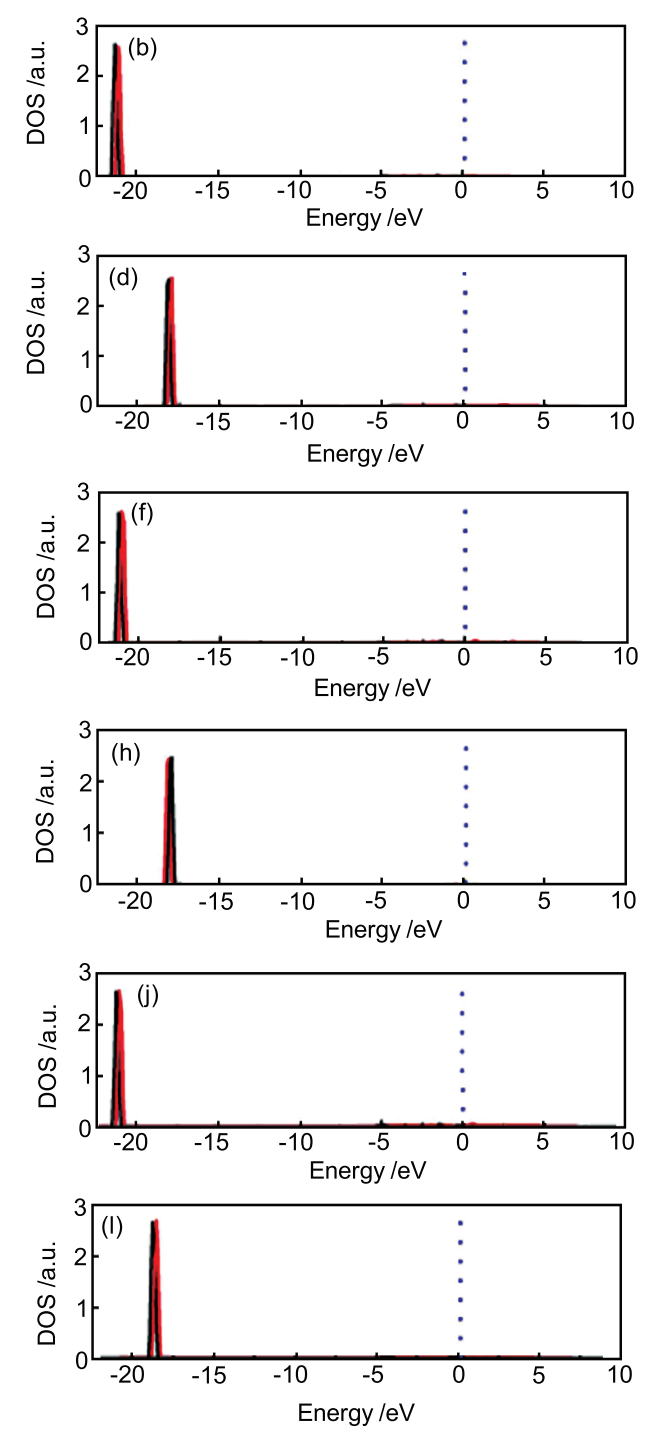

Fig. 4 The total local densities of states (DOS) of He atom and its nearest neighboring Fe atom: (a), (c), (e), (g), (i), and (k) correspond to the DOS of the Fe atoms where He atoms are at $O_{2 / /}, T_{2 / 3}, O_{3 / /}$, $T_{2 / 3}, O_{4 / /}$, and $T_{4 / 5}$, respectively; (b), (d), (f), (h), (j), and (l) are the corresponding DOS of He atoms, Fermi level is shifted to zero (dash vertical blue line) 
The total local densities of the states of He atom and its nearest neighboring $\mathrm{Fe}$ atom are plotted in Fig. 4(a), (c), (e), (g), (i) and (k) correspond to the DOS of the Fe atoms when He atoms at $O_{2 / /}, T_{2 / 3}$, $\mathrm{O}_{3 / /}, T_{2 / 3}, O_{4 / /}$, and $T_{4 / 5}$, respectively; Fig. 4(b), $(\mathrm{d}),(\mathrm{f}),(\mathrm{h}),(\mathrm{j})$ and (l) are the corresponding DOS of He atoms. Fermi level is shifted to zero (dash vertical blue line). From Fig. 4 it can be seen that for three OIS cases there are a little bit of overlapping of DOS of He atom and Fe atom at the energy level of $-21.25 \mathrm{eV}$. For three TIS cases there are less overlapping of DOS than the OIS ones. The overlapping position of the TIS cases is located at $-17.25 \mathrm{eV}$. Thus the overlapping DOS produces bonding states at $-21.25 \mathrm{eV}$ for the three OIS cases and at $-17.25 \mathrm{eV}$ for the three TIS cases. From the results of partial DOSs it is known that bonding states are formed by d electrons of $\mathrm{Fe}$ atom and s electrons of He atom. In such a Fe-He system their electrons at the range of energy from about $-7.5 \mathrm{eV}$ to Fermi level (see Fig. 4) are the ones to occupy anti-bonding states (call the DOS anti-bonding states, because that there are not overlapping of the partial DOS between He atom and Fe atom at the energy range). From Fig. 4 it can be seen that in the Fe-He system the number of electrons at the bonding states is far less than ones at anti-bonding states. In this situation the farther the bonding states are apart from the anti-bonding states filled with electrons, the more unstable the $\mathrm{He}$ atom is. In the OIS cases the bonding states are $5 \mathrm{eV}$ more apart from the electron-filled ant-bonding states than ones in the TIS cases. Thus He atom in the OIS cases is more un-stable than one in TIS cases. Similar situations can also be seen in the cases of $\mathrm{He}$ atom at $O_{5 / /} \rightarrow T_{5 / 6}$ and not be shown here. Similarly, when He impurity is relaxing into vacuum gap, such a situation can be also found. The character of the energy difference between bonding state and anti-bonding state is similar to that of the potential difference between two bells at a dumbbell "standing" vertically. The dumbbell with the two bells more apart from each other is more unstable than the one with the two bells approaching closely each other. In fact, it is worthy to note that although the energy levels of $\mathrm{He}$ at three unstable OIS sites are all lower than ones of He at the three corresponding stable TIS sites (shown in Fig. 4), the total energy of the three unstable cases are higher than ones of their respective corresponding stable cases. Thus the He at the OIS sites is more unstable than the $\mathrm{He}$ atom at the corresponding TIS sites.

Form the figure it can also be seen that the DOS over Fermi level in the three OIS cases increases more rapidly than the one in the corresponding TIS cases. A more rapid increase of DOS over Fermi level means that more electrons might be excited into the levels over Fermi level if the system is disturbed slightly by external factors, such as temperature, strain, pressure, etc. The more occupancy space of excited elec- trons indicates the more opportunities to undergo a transition of atom configuration. Thus it is seen that due to the imbalance distribution of deformation charge densities around the He atom at octahedral site $O_{n / /}$ the He atom undergoes a transition and is relaxed into slightly more stable tetrahedral site $T_{n / n+1}$, as shown in Fig. 3.

Finally, atomic magnetic moment and vacancy formation energy of the surface system are also calculated and listed in Table 1. From the table it can be seen that the magnetic moment of Fe atom changes with its position at surface atomic layer. In layer 1 the magnetic moment is the largest one, In layer 3 is the larger one, in layer 2 the magnetic moment is less than one in layer 1 and 3 , but greater than in layer 4, layer 5 , and in bulk. The magnetic moment in layer 4 and layer 5 are less than one in bulk. The magnetic moment of the Fe atom in the first three topmost SALs is greater than one in bulk. The formation energy of monovacancy is calculated to be $0.33,1.56,2.04,2.02$ and $2.11 \mathrm{eV}$ from the first to the 5th surface atomic layer.

\section{Conclusions}

First principle calculation is used to investigate the properties of various kinds of single point defects on (001) surface and bulk of bcc Fe. It is found that for the clean surface system the magnetic moments of Fe atom at the 4th and 5th topmost layers are less than those in bulk, while the magnetic moment in the first three topmost layers is greater than that in bulk. It is also found that there are three cases for stability of a He atom as an impurity at the surface. All of the He atom point defects in the first topmost SAL and some in the second SAL are unstable and relax into vacuum gap. In the other surface layer He atom at octahedral interstitial site with two nearest neighboring Fe atoms along horizontal direction is unstable and is relaxed into a more stable tetrahedral interstitial site, while He atom at octahedral interstitial site with two nearest neighboring Fe atoms along vertical direction is stable. He atom at tetrahedral interstitial site is stable. The formation energy of the point defects is found to be reduced with the change of the position of the point defects from the topmost surface atomic layer to bulk-like atomic layer. The formation energies of the monovacancy in the first to the 5th SALs are calculated to be equal to $0.33,1.56,2.04,2.02$ and $2.11 \mathrm{eV}$, respectively. From the topmost SAL to bulk-like atomic layer the formation energies increase gradually. This indicates that all kinds of the point defects considered have great trend to aggregate to the topmost SAL. The un-stability of the He atom at the surface system of bcc Fe is explained by deformation mechanism of charge densities and electron densities of the states. Moreover, it is found that the electronic cloud around $\mathrm{He}$ atom in the $\mathrm{He}-\mathrm{Fe}$ system is reduced. This may be helpful to form a weak-link 
between the two He atoms. If it is true, this might be a reason that in bcc Fe He atoms form He bubble. Of course, the un-stability, as illustrated and shown here, and the positive formation energy, as calculated in present work, of He impurity in bcc Fe could be greatly helpful to form a He bubble.

\section{Acknowledgements}

This work was supported by National Basic Research Program of China (No.2007CB209803), the National Natural Science Foundation of China (No.51231002) and the Applied Basic Research Program from Hebei Province.

\section{REFERENCES}

[1] R. Schaublin and N. Baluc, Nucl. Fusion 47 (2007) 1690.

[2] D.A. Terentyev, T.P.C. Klaver, P. Olsson, M.C. Marinica, F. Willaime, C. Domain and L. Malerba, Phys. Rev. Lett. 100 (2008) 145503.

[3] D.H. Xu and B.D. Wirth, J. Nucl. Mater. 386-388 (2009) 395

[4] C.C. Fu and F. Willaime, Phys. Rev. B 72 (2005) 064117.

[5] T. Seletskaia, Y. Osetsky, R.E. Stoller and G.M. Stocks, Phys. Rev. Lett. 94 (2005) 046403.

[6] C. Domain, J. Nucl. Mater. 351 (2006) 1.

[7] T. Seletskaia, Y. Osetsky, R.E. Stoller and G.M. Stocks, J. Nucl. Mater. 351 (2006) 109.

[8] J. Cai and M.R. Philpott, Comput. Mater. Sci. 30 (2004) 358.

[9] G. Kresse and J. Hafner, Phys. Rev. B 47 (1993) 588.
[10] G. Kresse and J. Furthmuller, Comput. Mater. Sci. 6 (1996) 15.

[11] W. Kohn and L. Sham, Phys. Rev. A 140 (1965) 1133.

[12] K. Laasonen, A. Pasquarello, R. Car, C. Lee and D. Vanderbilt, Phys. Rev. B 47 (1992) 10142.

[13] G. kresse, Ph.D. Thesis, Technische Universitat Wien, 1993.

[14] D. M. Bylander, L. Kleinman, and S. Lee, Phys. Rev. B 42 (1990) 1394.

[15] P. Pulay, Chem. Phys. Lett. 73 (1980) 393.

[16] R. Car and M. Parrinello, Phys. Rev. Lett. 55 (1985) 2471.

[17] D. Vanderbilt, Phys. Rev. B 41 (1990) 7892.

[18] G. Kresse and J. Joubert, Phys. Rev. B 59 (1999) 1758.

[19] J.P. Perdew and A. Zunger, Phys. Rev. B 23 (1981) 5048.

[20] J.P. Perdew, J.A. Chevary, S.H. Vosk, K.A. Jackson, M.R. Pederson, D.J. Shingh and C. Fiolhais, Phys. Rev. B 46 (1992) 6671.

[21] J.D. Pack and H.J. Monkhorst, Phys. Rev. 16 (1977) 1748.

[22] T. Berdot, A. Hallal, L.T. Bismaths, L. Joly, P. Dey, j. Henk, M. Alouani and W. Weber, Phys. Rev. B 82 (2010) 172407.

[23] D.H.R. Fors and G. Wahnstrom, Phys. Rev. B 82 (2010) 195410.

[24] X.T. Zu, L. Yang and F. Gao, Phys. Rev. B 80 (2009) 54104.

[25] L. Zhang, X.L. Shu, S. Jin, Y. Zhang, and G.H. Lu, J. Phys.: Condens. Matter. 22 (2010) 375401.

[26] C. Kittel, Introduction to Solid State Physics, 6th ed., Wiley, New York, 1986, p.521. 